\title{
Die Psychiatrie ist ein medizinisches Fach
}

\author{
Undine E. Langa, Kaspar Aebi ${ }^{b}$ \\ a Prof. Dr. med, Ordinaria für Psychiatrie der Universität Basel, Klinikdirektorin Erwachsenenpsychiatrie und Privatklinik, Universitäre Psychiatrische Kliniken \\ Basel (UPK), Mitglied FMH; ${ }^{b}$ Facharzt für Psychiatrie und Psychotherapie und für Allgemeinmedizin, Co-Präsident Kommunikation der Verbindung der \\ psychiatrisch-psychotherapeutisch tätigen ÄrztInnen der Schweiz (FMPP)
}

\section{Es ist normal, dass körperliche Erkrankungen zu psychischen Diagnosen führen}

Aktuelle epidemiologische Studien besagen, dass 50\% der Menschen im Lauf ihres Lebens eine psychiatrische Diagnose haben, in einer internationalen WHOStudie zeigte sich, dass etwa ein Drittel der PatientInnen, die zu ihrem Hausarzt gehen, explizit psychische Symptome äussern; wenn man sie aktiv befragt, erfüllen weitere 10\% die Diagnosekriterien einer psychischen Erkrankung [1].

Ein grosser Teil der Menschen, die in Allgemeinkrankenhäusern behandelt werden, hat entsprechend eine

\section{Résumé}

Les affections psychiques touchent souvent les personnes malades physiquement. II est essentiel de traiter leurs symptômes psychiques, car le psychisme influence le déroulement et le pronostic de la maladie physique.

Les symptômes psychiques sont souvent le premier signe d'une maladie physique sévère. Dans l'ensemble, les médicaments psychiatriques sont en moyenne d'une efficacité comparable à celle d'autres médicaments et agissent pour certains sur les symptômes physiques de même qu'une partie des médicaments de médecine interne agit sur le psychisme. Le traitement le plus efficace est celui associant mesures médicamenteuses et psychothérapeutiques. La psychothérapie est aujourd'hui tout aussi efficace que le pontage coronarien, par exemple. Ces dernières années, l'attention scientifique dont elle est l'objet, son importance dans la thérapie et les spécialisations qu'elle propose se sont renforcées. Le traitement psychothérapeutique de symptômes physiques est couronné de succès. Un rapprochement plus étroit entre la psychiatrie et les autres disciplines médicales serait avantageux pour les deux parties et surtout pour les patientes et patients concernés. Une équivalence générale de la médecine somatique et psychiatrique dans la formation médicale ainsi que dans les soins stationnaires et ambulatoires est indispensable pour atteindre cet objectif. L'intégration de la prise en charge psychiatrique dans les hôpitaux, universitaires et autres, avec des parcours de soins interdisciplinaires courts adaptés est nécessaire. akute psychische Störung. Bis zu 50\% der Menschen mit einem Diabetes leiden unter einer Depression, bis zu 30\% derjenigen, die einen Schlaganfall erlitten haben, bis zu 30\% der Menschen mit Schilddrüsenerkrankungen, bis zu 40\% der onkologischen Patienten, bis zu 30\% derjenigen mit einer Herz-Kreislauf-Erkrankung und bis zu 30\% der neurologischen Patienten [2]. Bei 10-30\% der älteren Patienten, die in ein Allgemeinkrankenhaus eintreten, liegt ein Delir vor, und während der Behandlung entwickeln ca. 30\% der über 70-Jährigen und bis zu 60\% der postoperativen Patienten ein Delir [3]. Bei ca. 14\% der internistischen und chirurgischen Patienten liegt die Diagnose einer Alkoholabhängigkeit vor [4]. Es ist also häufig, dass psychische Erkrankungen bei Menschen vorkommen, die körperlich krank sind.

De facto führen psychische Erkrankungen umgekehrt ebenfalls zu einem stark erhöhten Risiko für körperliche Erkrankungen und verschlechtern bei bereits bestehenden körperlichen Krankheiten die Prognose. So verdoppelt sich das Risiko für Diabetes, Demenz, Epilepsie, Herzkreislauf- oder Krebserkrankungen oder M. Parkinson, wenn eine Depression vorliegt, und die Symptome, die Prognose und Mortalität verschlechtern sich, wenn die Depression unbehandelt bleibt $[2,5]$. Psychische Nebendiagnosen in Schweizer Akutspitälern verdoppelten die Aufenthaltsdauer, die Rehospitalisierungsrate, die Mortalitätsrate und erhöhen den ökonomischen Ressourcenaufwand um ein Drittel [6]. Menschen, die unter einer schweren psychischen Erkrankung leiden, haben u.a. deshalb im Vergleich zur Allgemeinbevölkerung eine bis zu 15 Jahre verkürzte Lebenserwartung [7].

Depressive Erkrankungen gehen mit Veränderungen kardiovaskulärer, entzündlicher und metabolischer Risikofaktoren einher, sie verändern beispielsweise Cortisol, CRP, Cystein, Homocystein, Adiponektin, Gewicht, Blutgerinnung, glykiertes Hämoglobin, Leptin, Interferon Gamma, HDL, Interleukine, IGF, LDL, TNFalpha, Troponine und Triglyceride [2]. Antidepressiva haben teilweise eine prophylaktische und therapeuti- 
sche Wirkung bei onkologischen Erkrankungen, Diabetes, Schlaganfall, Entzündungen und Herzkreislauferkrankungen [5]. Umgekehrt haben internistische Medikamente, i.e. Statine, Aspirin, NSAIDs, Pioglitazon, Celecoxib und Minocyklin, eine therapeutische Wirkung bei psychiatrischen Erkrankungen [5].

Psychische Symptome sind häufig die ersten Hinweise auf eine schwere körperliche Erkrankung. In der psychiatrischen Times wurden in einem Übersichtsartikel 47 medizinische Erkrankungen aufgelistet, die als primäres Symptom Angst aufweisen [8]. Umgekehrt wurde in der Übersichtsarbeit eine Liste von 30 Medikamentenkategorien aufgestellt, die Angstsymptome verursachen können [9]. Depressionen sind ebenfalls häufig Vorboten medizinischer Erkrankungen, die Jahre vor der Diagnose auftreten, das gilt insbesondere für Morbus Cushing, Schilddrüsenerkrankungen, Hyperparathyroidismus, Pankreas- und Lungenkarzinome, Herzinfarkt, Morbus Wilson und AIDS [9]. Viele Menschen, die psychiatrische Hilfe suchen, sind also medizinisch krank, und viele Menschen sind psychiatrisch krank und suchen medizinische Hilfe. So werden beispielsweise bis zu 80\% der Depressionen von Hausärzten behandelt, und eine Studie in der Notaufnahme des Montreal Heart Institute zeigte, dass ein Viertel der kardialen Patienten ausschliesslich unter Panikattacken litt. Wenn sich die Fächer Psychiatrie und Medizin voneinander entfernen, so führt das zu Fehldiagnosen und Nichtbeachtung von Komorbidität, was für die Patienten in beiden Fällen lebensbedrohlich sein kann.

\section{Psychiatrische Therapie ist hochwirksam}

Trotz kontroverser Debatten vor allem bezüglich der antidepressiven Behandlung und Demenzbehandlung kann man sagen, dass die psychiatrische Pharmakotherapie sehr erfolgreich ist und im Erfolg einer internistischen Therapie vergleichbar [10]. Einige pharmakotherapeutische Behandlungen wie etwa die Behandlung des Aufmerksamkeitsdefizitsyndroms, Trichotillomanie, Depression, Schlaflosigkeit, Methadonsubstitution, Essstörungen und Phobien zeigen sehr hohe Behandlungserfolge bei medikamentöser Therapie mit einer Effektstärke von 0,8 [10].

Eine Metaanalyse, die insgesamt 61 Metaanalysen, 852 Studien und 137126 Studienteilnehmer einbezieht, untersucht die Wirksamkeit von Psychotherapie und Pharmakotherapie bei 21 psychiatrischen Diagnosen [11]. Insgesamt lag die Wirksamkeit hier im Schnitt bei 0,5; sie war bei der psychotherapeutischen Behandlung mit 0,58 sogar noch etwas höher als bei der pharmakotherapeutischen Behandlung [11].
Für schwere depressive Episoden, chronisch depressive Patienten, rezidivierende Depressionen sowie ältere depressive Patienten sind statistisch signifikante additive Effekte einer Kombinationstherapie gegenüber einer alleinigen Psychotherapie oder einer alleinigen Pharmakotherapie nachgewiesen worden [12]. Patienten, die psychotherapeutisch behandelt werden, weisen im Vergleich zu Patienten, die nur pharmakotherapeutisch behandelt werden, signifikant geringere Rückfallraten auf [13].

Patienten weisen bei einer Kombinationstherapie aus Antidepressiva und Psychotherapie weiterhin eine höhere Medikamentencompliance auf, brechen weniger häufig die Behandlung ab, kooperieren besser, nehmen weniger zusätzliche Behandlungsangebote in Anspruch, zeigen eine höhere soziale Anpassung und erreichen längerfristig die günstigsten Ergebnisse hinsichtlich der Rezidivhäufigkeit [14].

In den letzten Jahren wurden unterschiedlichste Psychotherapieverfahren intensiv wissenschaftlich beforscht und haben sich als hochwirksam erwiesen, so ist etwa Psychotherapie bei Angsterkrankungen etwa vergleichbar wirksam wie eine Bypasschirurgie [10]. Im Rahmen der Weiterentwicklung der kognitiven Verhaltenstherapie wurden in den vergangenen zehn Jahren die Methoden der Akzeptanz- und Commitmenttherapie (ACT) und der Achtsamkeitsbasierten Kognitiven Therapie entwickelt.

ACT wurde auch bei verschiedenen chronischen körperlichen Erkrankungen untersucht und verbesserte Adhärenz, Symptome und Outcome bei Epilepsie, Multipler Sklerose, HIV, Diabetes, chronischem Schmerz sowie kardialen Patienten [15].

In den oftmals komplexen somato-psychischen und psychosomatischen Wechselwirkungen ist ein fundiertes medizinisches Wissen unerlässlich für eine sichere und qualitativ hochstehende Diagnostik und Behandlung sowie die enge Bindung der Psychotherapie an die Medizin, wie es in der Psychiatrie sein sollte.

\section{Komplementärmedizin und Prävention gewinnen an Bedeutung}

Prävention und Früherkennung werden eine grössere Rolle spielen, da das Auftreten von psychischen Erkrankungen einen immer wichtigeren Faktor in der Gesellschaft darstellen wird. In diesem Kontext zeigte sich in den letzten Jahren, dass bei sämtlichen psychischen Erkrankungen die Ernährung eine Rolle spielen könnte; eine Ernährungsumstellung bei einer klinischen Depression bewirkte beispielsweise einen bis zu 30\%igen Symptomrückgang [16]. Die Durchführung eines strukturierten und supervidierten körperlichen 
Trainings wird in den Behandlungsleitlinien sämtlicher psychischer Erkrankungen, aber insbesondere der Depression, empfohlen [17]. Lichttherapie gilt als Behandlung der ersten Wahl bei saisonal abhängigen Depressionen [18], hilft aber auch bei vielen anderen Störungen.

\section{Psychiatrische Therapie ist nicht teuer und lohnt sich}

In der EU werden die Kosten durch depressionsbedingten Absentismus am Arbeitsplatz auf ca. 75 Milliarden Euro pro Jahr geschätzt, Depressionen kosten damit den Arbeitgeber doppelt so viel wie etwa Herz-Kreislauf-Erkrankungen [19]. In der Schweiz liegen die Kosten für Depressionen am Arbeitsplatz bei ca. 8 Milliarden CHF pro Jahr [20]. Eine effektive Behandlung einer Depression in der Schweiz kostet im Schnitt ca. 10000 CHF und ist damit günstiger als die finanziellen Belastungen durch die Ausfälle am Arbeitsplatz, die durch eine - unbehandelte - Depression im Schnitt entstehen (ca. 13000 CHF) [20].

Eine europaweite Studie hat die ökonomische Effektivität eines Screenings am Arbeitsplatz untersucht und kommt zu dem Schluss dass die Früherkennung von Depressionen durch ein Screening-Instrument für einen Arbeitgeber kosteneffektiv ist, was sowohl für eine günstigere Pharmakotherapie als auch für eine teurere Psychotherapie gilt [19]. De facto war die Psychotherapieoption in dieser Studie zwar teurer als die Pharmakotherapie, zeigte aber über den Zeitraum von 27 Monaten eine bessere Kosteneffektivität, da sie die sogenannten QALYS (Quality Adjusted Life Years) positiver beeinflusste [19].

Vorschläge möglicher Modellprojekte für eine bessere Kooperation zwischen der Psychiatrie und anderen medizinischen Fächern

- Integration psychiatrischer Abteilungen in somatische Spitäler mit einer gemeinsamen (internistischen und psychiatrischen) interdisziplinären Leitung

- Erhöhung der internistischen bzw. allgemeinmedizinischen Kompetenz von psychiatrischen FachärztInnen oder etwa der psychiatrischen Kompetenz von Hausärzten mit beispielsweise gemeinsamen Stellenangeboten (Rotationsstellen) im Rahmen der ärztlichen Weiterbildung

Prof. Dr. Undine Lang

Universitäre Psychiatrische

Kliniken Basel (UPK)

Universität Basel

Wilhelm Klein-Str. 27

CH-4002 Basel

Tel. 0613255200

Undine.lang[at]upkbs.ch
Literatur

1 Ûstùn TB, Sartorius N. Mental Illness in General Health Care: An International Study. Chichester, UK: John Wiley \& sons; 1995.

2 Lang UE, Borgwardt S. Molecular mechanisms of depression: perspectives on new treatment strategies. Cell Physiol Biochem. 2013;31(6):761-77.

3 Mast van der RC, Roest FHJ. Delirium after cardiac surgery: Am critical review. J Psychosom Res. 1996;41:13-30.

4 Arolt V, Driessen M, Schürmann A. Häufigkeit und Behandlungsbedarf von Alkoholismus bei internistischen und chirurgischen Krankenhauspatienten. Fortschr Neurol Psychiatr. 1995;63:283-8.

5 Lang UE, Walter M. Depression in the Context of Medical Disorders: New Pharmacological Pathways Revisited. Neurosignals. 2017;25:54-73.

6 Tuch A. Somatisch-psychische Komorbidität in Schweizer Akutspitälern. Prävalenz und Inanspruchnahme. (Obsan Bulletin 1/2018). Neuchâtel: Schweizerisches Gesundheitsobservatorium.

7 Chang CK, Hayes RD, Perera G, Broadbent MTM, Fernandes AC, Lee WE, et al. Life Expectancy at Birth for People with Serious Mental Illness and Other Major Disorders from a Secondary Mental Health Care Case Register in London. PLoS One. 2011;6:e19590.

8 Dong Y, Noorani F, Vyas R, Balgobin C, Torres-Llenza V, Crone C. Managing Anxiety in the Medically Ill. Psychiatric Times. 2017;32.

9 Cosci F, Fava GA, Sonino N. Mood and Anxiety Disorders as Early Manifestations of Medical Illness: A Systematic Review. Psychother Psychosom. 2015;84:22-9.

10 Leucht S, Hierl S, Kissling W, Dold M, Davis JM. Putting the efficacy of psychiatric and general medicine medication into perspective: review of meta-analyses. Br J Psychiatry. 2012;200:97-106.

11 Huhn M, Tardy M, Spineli LM, Kissling W, Förstl H, Pitschel-Walz G, et al. Efficacy of Pharmacotherapy and Psychotherapy for Adult Psychiatric Disorders. A Systematic Overview of Metaanalyses. JAMA Psychiatry. 2014;71:706-15.

12 Cuijpers P, van Straten A, Warmerdam L, et al. Psychotherapy versus the combination of psychotherapy and pharmacotherapy in the treatment of depression: a meta-analysis. Depress Anxiety. 2009;26:279-88.

13 Simons AD, Murphy GE, Levine JL, Wetzel RD. Cognitive therapy and pharmacotherapy for depression. Sustained improvement over one year. Arch Gen Psychiatry. 1986;43:43-8.

14 Sutej I. Pharmakotherapie und Psychotherapie bei unipolarer Depression. Ist die kombinierte Behandlung einer Monotherapie überlegen? Zeitschrift für Psychiatrie, Psychologie und Psychotherapie. 2006;54:163-72.

15 Spatola CA, Manzoni GM, Castelnuovo G, Malfatto G, Facchini $\mathrm{M}$, Goodwin CL, et al. The ACTonHEART study: rationale and design of a randomized controlled clinical trial comparing a brief intervention based on Acceptance and Commitment Therapy to usual secondary prevention care of coronary heart disease. Health Qual Life Outcomes. 2014;12:22.

16 Jacka FN, O'Neil A, Opie R, Itsiopoulos C, Cotton S, Mohebbi M, et al. A randomised controlled trial of dietary improvement for adults with major depression (the 'SMILES' trial). BMC Med. 2017;15:23.

17 National Institute for Clinical Excellence (NICE). Depression: the treatment and management of depression in adults (update). Draft for consultation Feb 2009.

18 Lam RW, Gorman CP, Michalon M, et al. Multicenter, placebocontrolled study of fluoxetine in seasonal affective disorder. Am J Psychiatry. 1995;152:1765-70.

19 Gustavsson A, Svensson M, Jacobi F, et al. Cost of disorders of the brain in Europe 2010. Eur Neuropsychopharmacol. 2001;21:718-79.

20 Tomonaga Y, Haettenschwiler J, Hatzinger M, Holsboer-Trachsler E, Rufer M, Hepp U, et al. The economic burden of depression in Switzerland. Pharmacoeconomics. 2013;31:237-50.

\section{Interessenverbindungen}

Es liegt kein Interessenkonflikt der Autoren vor. 